\title{
Urine Iodine, Estrogen, and Breast Disease
}

\author{
Alexander E. Poor ${ }^{1 *}$, Bernard A. Eskin ${ }^{2}$, Christine Georgiadis ${ }^{1}$, Brian Hamzavi ${ }^{1}$, Ari D. Brooks ${ }^{1}$ \\ ${ }^{1}$ Department of Surgery, Drexel University College of Medicine, Philadelphia, USA; ${ }^{2}$ Department of Obstetrics and Gynecology, \\ Drexel University College of Medicine, Philadelphia, USA. \\ Email: *alexander.poor@gmail.com
}

Received October $14^{\text {th }}, 2012$; revised November $16^{\text {th }}, 2012$; accepted November $25^{\text {th }}, 2012$

\begin{abstract}
Introduction: We sought to explore iodine status (expressed as urine iodine excretion) in a group of women with a variety of breast diseases to characterize the relationship between iodine and breast physiology. Methods: Demographic and clinical data were recorded and random urine iodine levels were checked in 415 euthyroid patients over 5 years during clinic visits. Results: Pre-menopausal women excreted less iodine in their urine than post menopausal women. Post menopausal women who took hormone supplementation had an excretion level similar to pre menopausal women. Women with breast cancer excreted less iodine than those without, and iodine excretion increased after treatment with aromatase inhibitors. Conclusions: These results demonstrate that there is a relationship between estrogen status and iodine excretion in euthyroid women. This suggests that there may be a threshold for total body iodine required for prevention of breast disease.
\end{abstract}

Keywords: Breast Cancer; Iodine; Iodide; Estrogen

\section{Introduction}

There is a great deal of epidemiologic, clinical and basic science data to support the fact that iodine plays a role in breast physiology [1-7]. Iodine is essential for lactation and there is an association between thyroid disease and breast disease [8-10]. Animal models have suggested that iodine deficiency increases the incidence of breast dysplasia and lowers the threshold for breast carcinogenesis [2,11-14]. Several clinical trials have looked at iodine supplementation to treat fibrocystic disease and breast pain $[12,15]$.

While the World Health Organization has established a normal daily iodine intake to avoid goiter [16] and the National Institutes of Health has determined what constitutes a normal urine iodine level [17], changes in these parameters have not been reported for women with breast disease. It is well known that variations in diet can cause fluctuations in urinary iodine levels, but only in chronic conditions. Knowing that iodine is important in breast physiology, we sought to explore iodine status (expressed as urine iodine excretion) in a group of euthyroid women with a variety of breast diseases.

\section{Study Design and Methods}

The Drexel University College of Medicine Institutional Review Board approved our study protocol. In adherence

\footnotetext{
"Corresponding author.
}

to this protocol, a total of 415 patients presenting to the Drexel University Women's Health Program provided consent to participate in the study and for the results of the study to be published and were enrolled in the study between 2004 and 2009. Patients were screened and were excluded for any history of thyroid disease, surgery, or thyroid medications. Random urine iodine levels were checked during clinic visits and patient data including presentation, gynecologic history, medications, breast cancer risk factors, diagnosis, and treatment were recorded. Many women gave urine samples on 2 or more occasions, often before and after treatment for breast cancer. Some healthy volunteers gave 4 weekly specimens for our menstrual cycle study.

Urine specimens were stored in a freezer at $-20^{\circ} \mathrm{C}$ until batches were processed using the ion-selective electrode assay (ISE) method. This procedure involves isolation of urinary iodine by anion exchange chromatography that is then quantified by measuring the electromotive force generated at an iodide-selective electrode. The details of this procedure have been previously described [18].

Data were stored on a secure server, de-identified, and analyzed using SPSS software. Chi square, t-test, paired $t$-test and ANOVA were used where appropriate.

\section{Results}

Between 2004 and 2009, 415 patients were enrolled in 
the study with 451 evaluable urine specimens obtained. The demographics are reported in Table 1 with the patients separated into 2 groups based on menopausal status. The two populations are significantly different in ethnicity and prevalence of cancer. Many patients gave more than one sample during the study period, usually after a cancer diagnosis had been obtained and treatment had begun.

In order to explore the relationship between hormone status and urine iodine levels, the data was evaluated in two ways. First the urine iodine levels were compared in relation to reported menopausal state (Figure 1). Then women who were using hormone replacement therapy were moved from the post menopausal group to the pre menopausal group (high estrogen). It is clear from the graph that there was a trend towards lower urine iodine excretion in women labeled as "pre menopause"/"high" vs. "post menopause"/“low" estrogen state. The results of the analysis are reported for all women in the group, and then women without a cancer diagnosis are analyzed separately.

Looking at the urine iodine excretion in patients diagnosed with cancer vs that seen in other women, there is a trend towards lower urine iodine excretion in pre menopausal women diagnosed with cancer. When the same analysis is done for high vs low estrogen state (Figure 2), the difference becomes more apparent in the high estrogen patients.

In an effort to explore the link between hormone status, malignancy, and urinary iodine excretion a little further, we looked at our patients who gave us a second sample after they had undergone treatment for their cancer and compared them to other women who gave us multiple samples over time with no cancer diagnosis. In this analysis we identified 15 women (11 post menopausal) who gave one sample before and at least one after treatment over a median interval of 12 months (range 1 - 29). We also identified 7 women (5 post menopausal) with benign breast complaints with at least 2 samples (over a median interval of 9 months (range 4 - 11). In benign patients, the mean starting iodine level was $140 \mu \mathrm{g} / \mathrm{l}$ and the mean follow up level was $150 \mu \mathrm{g} / \mathrm{l}$ with a mean difference of $10 \mu \mathrm{g} / \mathrm{l}$. In patients with a cancer diagnosis, the starting urine iodine level was $120 \mu \mathrm{g} / \mathrm{l}$ and increased to mean of $230 \mu \mathrm{g} / 1$ with a mean difference of $120 \mu \mathrm{g} / \mathrm{l}$. $(\mathrm{p}<0.05)$.

Looking specifically at the urine iodine levels in women with cancer we found that in both pre and post menopausal women, there was a trend towards lower urinary iodine levels in women with estrogen receptor (ER) positive cancers (Figure 3). The results are similar for progesterone receptor (PR) status in the group with PR positive pre menopausal women trending to have lower urinary iodine excretion than those with PR positive tumors.

Figure 4 is a graph representing the urinary iodine excretion for women with various estrogen states. The urinary iodine levels are lowest for pre menopausal women

Table 1. Demographics and diagnoses of pre and postmenopausal patients.

\begin{tabular}{lcc}
\hline & Pre-menopausal & Post-menopausal \\
\hline Patients & 212 & 173 \\
Median Age (yr) & 37 & 58 \\
Total Specimens & 253 & 196 \\
Caucasian & $35 \%$ & $24 \%$ \\
African American & $49 \%$ & $67 \%$ \\
Hispanic & $7 \%$ & $5 \%$ \\
Asian & $9 \%$ & $3 \%$ \\
Not Reported & $0 \%$ & $1 \%$ \\
Number with CA & 22 & 68 \\
\multicolumn{1}{c}{ DCIS } & 3 & 19 \\
\multicolumn{1}{c}{ Invasive } & 19 & 49 \\
\hline
\end{tabular}

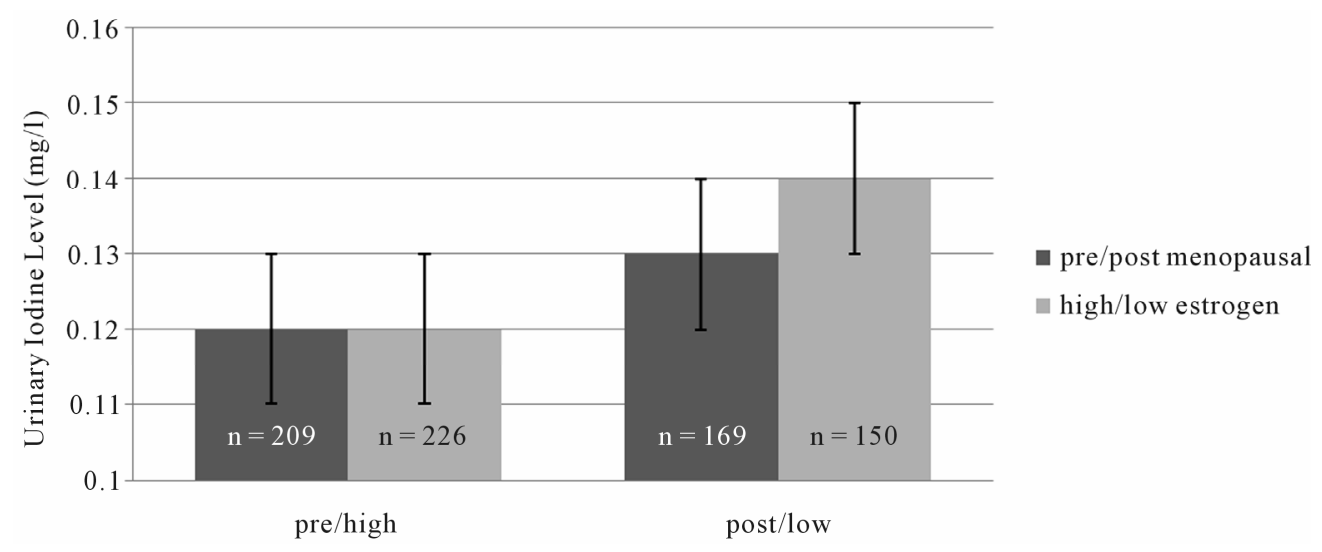

Figure 1. Trend towards lower urinary iodine excretionin high estrogen states $(p=n s)$. 
with cancer $(\mathrm{n}=22,100 \pm 10 \mu \mathrm{g} / \mathrm{l})$. Pre menopausal women without a diagnosis of cancer and on no birth control pills excrete slightly more urinary iodine $(\mathrm{n}=122$, $120 \pm 10 \mu \mathrm{g} / \mathrm{l})$. Post menopausal women with cancer have a slightly higher mean urine iodine $(\mathrm{n}=51,130 \pm$ $10 \mu \mathrm{g} / \mathrm{l})$. Women taking Tamoxifen or another selective estrogen receptor modulator have a mean urine iodine level of $130 \pm 20 \mu \mathrm{g} / \mathrm{l}(\mathrm{n}=20)$. Post menopausal women without cancer and not on hormone replacement have next highest urine iodine levels $(\mathrm{n}=105,140 \pm 20 \mu \mathrm{g} / \mathrm{l})$. The mean urine iodine level for women on aromatase inhibitors is $150 \pm 10 \mu \mathrm{g} / \mathrm{l}(\mathrm{n}=16)$. Pre menopausal women on birth control pills without a cancer diagnosis have the highest urine iodine level $(\mathrm{n}=28,170 \pm 10$ $\mu \mathrm{g} / \mathrm{l})$.

Hormone status and urine iodine levels were explored using just the women without cancer in Figure 5. For women not on birth control pills, in the first week during menses the urine iodine level is $60 \mu \mathrm{g} / \mathrm{l}(\mathrm{n}=3)$ rising to a mean of $110 \mu \mathrm{g} / \mathrm{l}(\mathrm{n}=5)$ in week two. Week three rises to $190 \mu \mathrm{g} / \mathrm{l}(\mathrm{n}=4)$ and then drops again in week 4 to 110 $\mu \mathrm{g} / \mathrm{l}(\mathrm{n}=3)$ at the end of the cycle. In comparison, the mean urine excretion in our 8 pregnant women was 110 $\mu \mathrm{g} / \mathrm{l}$. As stated above, the mean urine iodine excretion was $140 \mu \mathrm{g} / \mathrm{l}$. This correlates with expected serum estrogen levels as seen in the same figure. For women on birth control pills, the trend was slightly different, with a menstruation week mean excretion of $210 \mu \mathrm{g} / \mathrm{l}(\mathrm{n}=8)$, dropping to $120 \mu \mathrm{g} / \mathrm{l}(\mathrm{n}=5)$ in week 2 and then rising again in weeks $3(160 \mu \mathrm{g} / \mathrm{l}, \mathrm{n}=9)$ and $4(180 \mu \mathrm{g} / \mathrm{l}, \mathrm{n}=7)$. Most birth control pills provide steady levels of progesterone in weeks 1 - 3 with a withdrawal in week 4.

Patients diagnosed with benign breast pain had a lower urinary iodine excretion $(\mathrm{n}=30,100 \mu \mathrm{g} / \mathrm{l})$ than other women with benign disease $(\mathrm{n}=257,130 \mu \mathrm{g} / \mathrm{l})$. This did not change when evaluated by menopausal status. Pre menopausal patients who smoke had lower urine iodine levels $(\mathrm{n}=49,90 \mu \mathrm{g} / \mathrm{l})$ versus non-smokers $(\mathrm{n}=198,130$ $\mu \mathrm{g} / \mathrm{l})$.

\section{Discussion}

We have shown that pre menopausal women excrete less iodine in their urine than post menopausal women. Further, we have shown that post menopausal women who

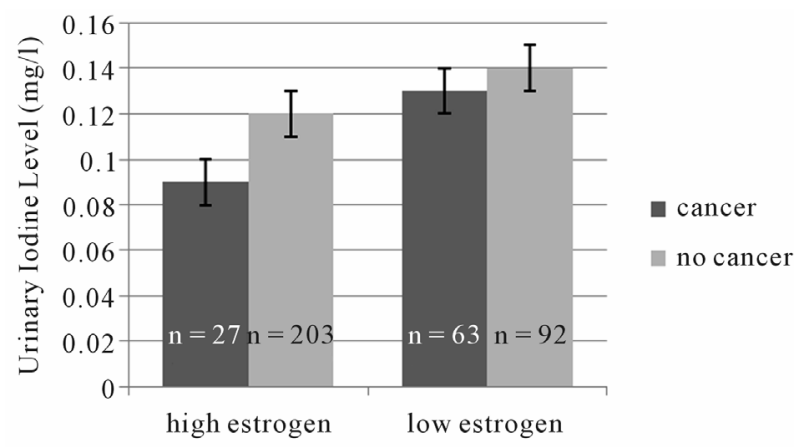

Figure 2. Trend towards lower urinary iodine excretion in women with high estrogen and cancer $(p=n s)$.

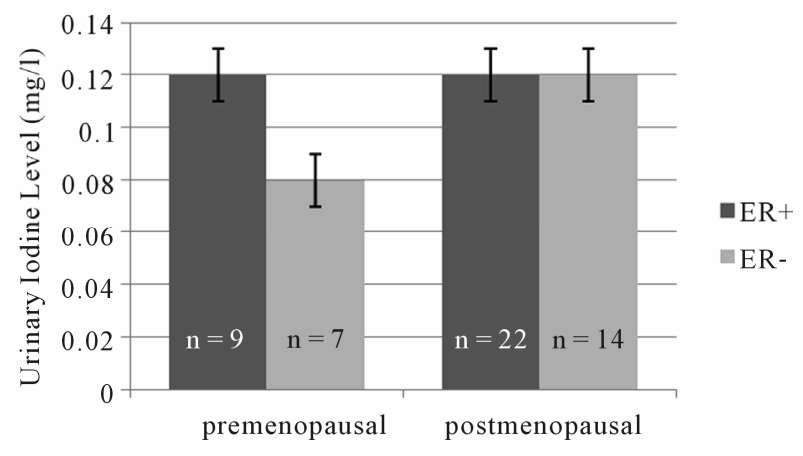

Figure 3. Trend towards lower urinary iodine excretion in women with ER-tumors (p = ns).

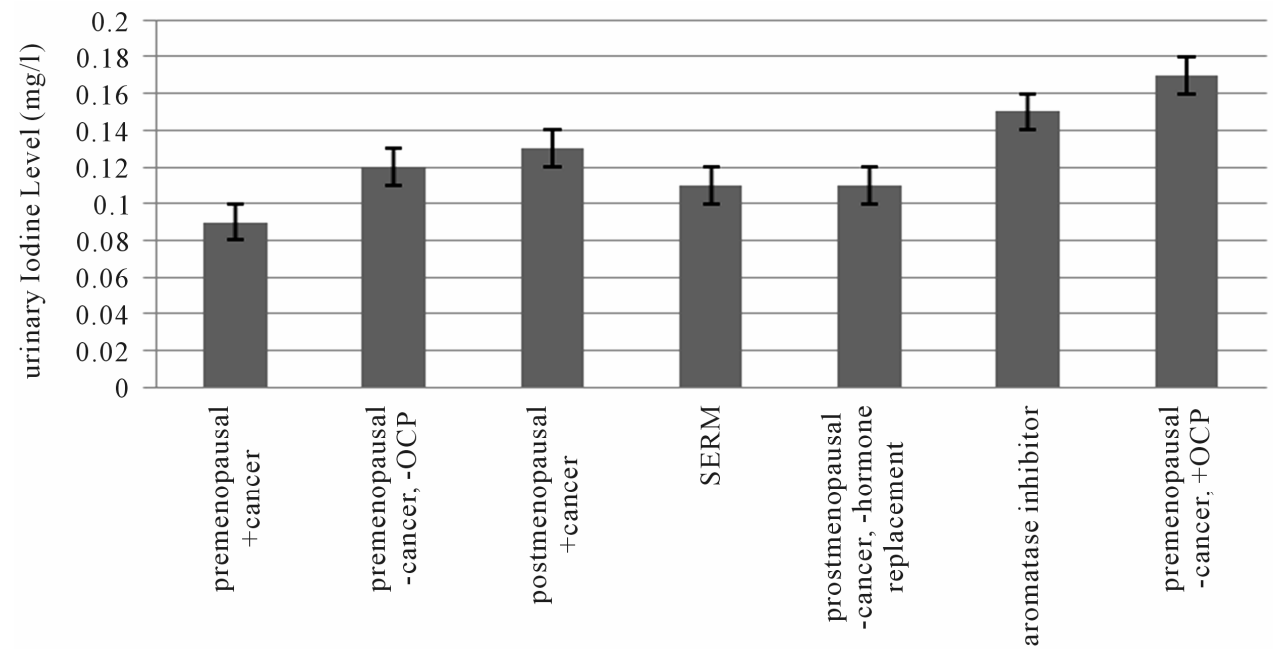

Figure 4. Urinary iodine excretion in various estrogen states $(p<0.05)$. 


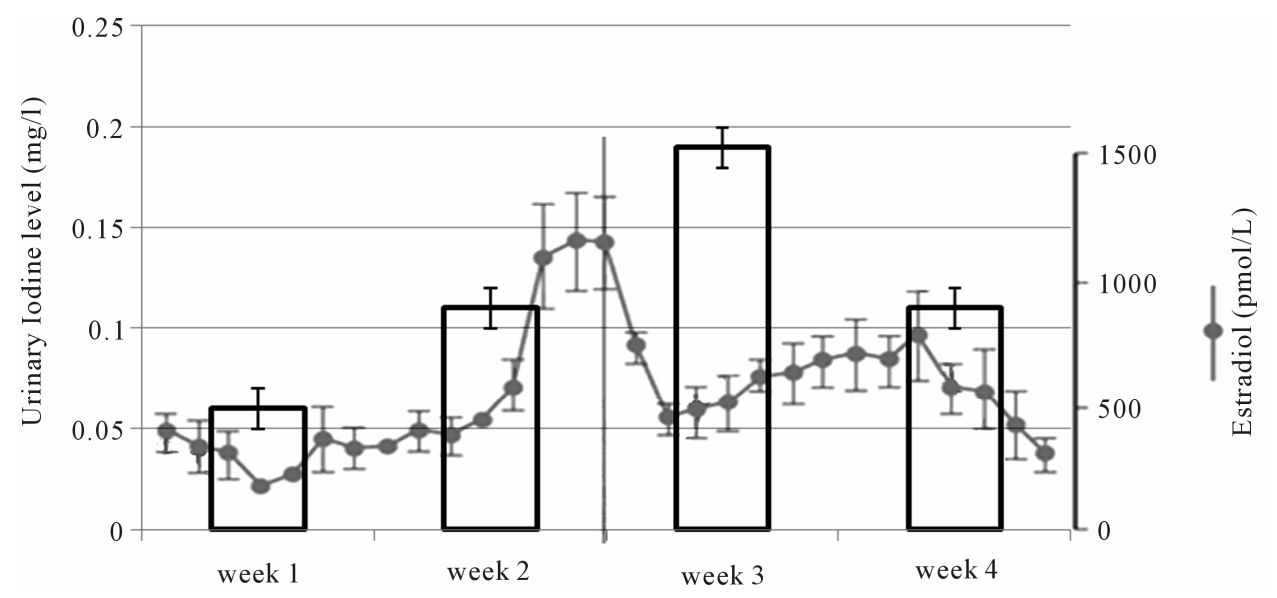

Figure 5. Urinary iodine excretion and estrogen level during the menstrual cycle (nonpregnant women without cancer).

take hormone supplementation have an excretion level similar to normal pre menopausal women. In the pre menopausal women who were tested during a regular menstrual cycle we noted fluctuation in the urinary iodine excretion in association with hormone changes. There are several possible explanations for this finding: 1) estrogen-dependent changes in the iodine binding proteins; 2) estrogen increases renal iodine excretion; 3) changes in iodine uptake by estrogen responsive organs under circumstances of adequate estrogen levels [19].

There is little data to evaluate the first two possible explanations, but there is a growing amount of evidence that the third explanation plays a role in the observed estrogen-dependent fluctuations in iodine excretion. The organ most widely discussed with regards to iodine metabolism, the thyroid, is estrogen-responsive. Although the thyroid is primarily concerned with iodine and thyroid hormone regulation, estrogen seems to play an important basic and clinical role in thyroid disease. Thyroid disease has at least a 3:1 predominance in women and certain thyroid cancers are common in women of reproductive age and rare in post menopausal women and men [20]. With regards to normal thyroid tissue, estrogen receptors are present in the thyroid gland [21] and estrogen induces thyroid cell growth in-vitro [10]. In this way, the thyroid is similar to the breast: a highly hormone-responsive, estrogen receptor (ER) positive organ whose growth is stimulated by both high estrogen and low iodine states. It should be noted that this description might also be applied to the endometrium. But the breast plays an important role in molecular iodine $\left(\mathrm{I}_{2}\right)$ metabolism independent of either the thyroid or endometrium.

Numerous studies have demonstrated that while iodide $\left(\mathrm{I}^{-}\right)$is metabolically more effective in the thyroid gland, iodine $\left(I_{2}\right)$ is more active in the breast $[3,8,22]$. Furthermore, iodine is required to maintain normal physiologic homeostasis $[4,13]$ and increased iodine uptake by the breast is critical during lactation [23-25]. Normal breast function requires, at times, increased iodine uptake that is likely hormone-mediated.

The relationship between iodine and estrogen metabolism also involves iodine affecting estrogen signals in the breast independent of the thyroid. For example, iodine diminishes dysplasia in clinical trials and breast tumor growth in rats, more effectively than iodide ( $\left.\mathrm{I}^{-}\right)$and thyroxine $\left(T_{4}\right)[8,19,26]$. In fact, thyroxine $\left(T_{4}\right)$ failed to diminish tumor growth in iodine-deficient rats following N-Nitroso-N-methylurea or 7,12-Dimethylbenz(a) anthracene administration [8].

Few studies had described iodine's mechanism of action or its target pathways. Recently researchers have suggested that iodine inhibits breast cancer growth via cyclin-independent apoptosis; however there is evidence that a different pathway is involved. We analyzed the effects of iodine on global gene expression in estrogen responsive MCF-7 breast cancer cell line. Microarray analysis and quantitative real time polymerase chain reaction (RT-PCR) indicated that iodine inhibits the expression of estrogen-responsive genes TFF1 and WISP2 [27]. Our data further provided three potential mechanisms to explain the observed decrease in estrogen response. First, iodine treatment results in decreased ER $\alpha$ mRNA levels; second, iodine up-regulates genes involved in estrogen metabolism (CYP1A1, CYP1B1, and AKR1C1), and finally, iodine decreases Cyclin D1 (a competitive inhibitor of BRCA1) mRNA levels which may functionally permit BRCA1 inhibition of estrogen responsive transcription. Thus the interaction between iodine and estrogen signaling may inhibit breast cancer growth by affecting an intermediate, perhaps the estrogen receptor system.

This study serves as an initial evaluation of the association between breast disease states, estrogen status, and urine iodine excretion. The results must be taken in light of certain limitations. First, these were random spot urine samples, while 24-hour urine, and 1st morning void sam- 
ples might be a more accurate reflection of urine excretion, urine iodine excretion is relatively stable throughout the day and random urines have been shown to be reflective of overall urine excretion. Second, serum estrogen, progesterone, follicle stimulating hormone (FSH) and luteinizing hormone (LH) were not measured in this study, this information will be essential in any later confirmatory studies. Third, fluctuations in dietary iodine intake were not recorded, and this would be important as well. In the evaluation of urine iodine changes over time, only a small percentage of the total number of women tested had repeat specimens. Overall, this report serves as the first investigation of the association between estrogen status, breast disease and iodine levels.

\section{Conclusions}

The present results demonstrate again that there is a relationship between estrogen status and iodine excretion and suggest that there is a threshold for iodine required for maintenance of normal breast function in pre menopausal women. Thus, urine iodine levels may be useful in breast cancer risk assessment among these patients.

Future research will examine the mechanisms by which iodine inhibits the estrogen pathway, down-regulates CCND1 (thought to play a role in the development of tamoxifen resistance), and up-regulates (CYP1A1) and other estrogen metabolizing enzymes, which may allow Iodine supplementation to work synergistically with aromatase inhibitors in the treatment/prevention of breast cancer.

\section{Authors' Contributions}

$\mathrm{BE}$ and $\mathrm{AB}$ conceived of the study, participated in its design and coordination, and helped to draft the manuscript. AP performed the statistical analysis and helped to draft the manuscript. CG and $\mathrm{MH}$ performed the data acquisition. All authors read and approved the final manuscript.

\section{Acknowledgements}

This work was supported in part by the Beukenkamp Endowment for Prostate Cancer in Drexel University College of Medicine, Department of Surgery. We thank Dr. Guy Abraham, Optimox Corporation for conducting the blinded urine analysis assays.

\section{REFERENCES}

[1] B. A. Eskin, H. I. Jacobson, V. Bolmarich and J. A. Murray, "Breast Atypia in Altered Iodine States: Intracellular Changes," Senologia, Vol. 2, 1977, pp. 61-65.

[2] H. Funahashi, T. Imai, Y. Tanaka, et al., "Suppressive Effect of Iodine on DMBA-Induced Breast Tumor
Growth in the Rat," Journal of Surgical Oncology, Vol. 61, No. 3, 1996, pp. 209-213. doi:10.1002/(SICI)1096-9098(199603)61:3<209::AID-JS O9>3.0.CO;2-F

[3] P. Garcia-Solis, Y. Alfaro, B. Anguiano, et al., "Inhibition of N-Methyl-N-Nitrosourea-Induced Mammary Carcinogenesis by Molecular Iodine (I2) but Not by Iodide (I-) Treatment Evidence That I2 Prevents Cancer Promotion," Molecular and Cellular Endocrinology, Vol. 236, No. 1-2, 2005, pp. 49-57.

[4] B. A. Eskin, R. Shuman, T. Krouse and J. A. Merion, "Rat Mammary Gland Atypia Produced by Iodine Blockade with Perchlorate," Cancer Research, Vol. 35, 1975, pp. 2332-2339.

[5] P. S. Yang, T. L. Yang, C. L. Liu, C. W. Wu and C. Y. Shen, "A Case-Control Study of Breast Cancer in Taiwan-A Low-Incidence Area," British Journal of Cancer, Vol. 75, No. 5, 1997, pp. 752-756.

doi: $10.1038 /$ bjc. 1997.133

[6] K. Brown-Grant, "The Iodide Concentrating Mechanism of the Mammary Gland," Journal of Physiology, Vol. 135, No. 3, 1957, pp. 644-654.

[7] O. I. Alatise and G.N. Schrauzer, "Lead Exposure: A Contributing Cause of the Current Breast Cancer Epidemic in Nigerian Women," Biological Trace Element Research, Vol. 136, No. 2, 2010, pp. 127-139. doi:10.1007/s12011-010-8608-2

[8] B. A. Eskin, S. A. Murphey and M. R. Dunn, "Induction of Breast Cancer in Altered Thyroid States," Nature, Vol. 218, No. 5147, 1968, p. 1162. doi:10.1038/2181162a0

[9] P. R. K. Bhargav, A. Mishra, G. Agarwal, A. Agarwal, A. K. Verma and S. K. Mishra, "Prevalence of Hypothyroidism in Benign Breast Disorders and Effect of Thyroxine Replacement on the Clinical Outcome," World Journal of Surgery, Vol. 33, No. 10, 2009, pp. 2087-2093. doi:10.1007/s00268-009-0143-y

[10] A. Vivacqua, D. Bonofiglio, L. Albanito, et al., "17betaEstradiol, Genistein, and 4-Hydroxytamoxifen Induce the Proliferation of Thyroid Cancer Cells through the g Protein-Coupled Receptor GPR30," Molecular Pharmacology, Vol. 70, 2006, pp. 1414-1423. doi:10.1124/mol.106.026344

[11] B. V. Stadel, "Dietary Iodine and Risk of Breast, Endometrial, and Ovarian Cancer," Lancet, Vol. 307, No. 7965, 1976, pp. 890-891. doi:10.1016/S0140-6736(76)92102-4

[12] W. R. Ghent, B. A. Eskin, D. A. Low and L. P. Hill, "Iodine Replacement in Fibrocystic Disease of the Breast," Canadian Journal of Surgery, Vol. 36, No. 5, 1993, pp. 453-460.

[13] B. A. Eskin, D. G. Bartuska, M. R. Dunn, G. Jacob and M. B. Dratman, "Mammary Gland Dysplasia in Iodine Deficiency. Studies in Rats," JAMA, Vol. 200, No. 8, 1967, pp. 691-695. doi:10.1001/jama.1967.03120210077014

[14] B. A. Eskin, "Dietary Iodine and Cancer Risk," Lancet, Vol. 2, 1976, pp. 807-808. doi:10.1016/S0140-6736(76)90646-2

[15] J. H. Kessler, "The Effect of Supraphysiologic Levels of Iodine on Patients with Cyclic Mastalgia," Breast Journal, 
Vol. 10, No. 4, 2004, pp. 328-336. doi:10.1111/j.1075-122X.2004.21341.x

[16] WHO/UNICEF/ICIDD, "Recommended Iodine Levels in Salt and Guidelines for Monitoring Their Adequacy and Effectiveness," WHO, Geneva, 1996.

[17] K. L. Caldwell, R. Jones and J. G. Hollowell, "Urinary Iodine Concentration: United States National Health and Nutrition Examination Survey 2001-2002," Thyroid, Vol. 15, No. 7, 2005, pp. 692-699. doi:10.1089/thy.2005.15.692

[18] G. E. Abraham, J. D. Flechas and J. C. Hakala, "Measurement of Urinary Iodide Levels by Ion-Selective Electrode: Improved Sensitivity and Specificity by Chromatography on Anion-Exchange Resin," The Original Internist, Vol. 11, No. 4, 2004, pp. 19-32

[19] B. A. Eskin, C. E. Grotkowski, C. P. Connolly and W. R. Ghent, "Different Tissue Responses for Iodine and Iodide in Rat Thyroid and Mammary Glands," Biological Trace Element Research, Vol. 49, 1995, pp. 9-19. doi:10.1007/BF02788999

[20] L. C. Sakoda and P. L.Horn-Ross, "Reproductive and Menstrual History and Papillary Thyroid Cancer Risk: The San Francisco Bay Area Thyroid Cancer Study," Cancer Epidemiology, Biomarkers \& Prevention, Vol. 11, 2002, pp. 51-57.

[21] I. Lewy-Trenda, "Estrogen and Progesterone Receptors in Neoplastic and Non-Neoplastic Thyroid Lesions," Polish Journal of Pathology, Vol. 53, 2002, pp. 67-72.
[22] C. Aceves, B. Anguiano and G. Delgado, "Is Iodine a Gatekeeper of the Integrity of the Mammary Gland?" Journal of Mammary Gland Biology and Neoplasia, Vol. 10, No. 2, 2005, pp. 189-196. doi:10.1007/s10911-005-5401-5

[23] K. Brown-Grant, "The Iodide Concentrating Mechanism of the Mammary Gland," Journal of Physiology, Vol. 135, No. 3, 1957, pp. 644-654.

[24] K. Brown-Grant, "Iodinated Compounds in Milk after Radio-Iodide Administration," Biochimica et Biophysica Acta, Vol. 27, 1958, pp. 422-423. doi:10.1016/0006-3002(58)90358-5

[25] B. A. Eskin, J. A. Parker, J. G. Bassett and D. L. George, "Human Breast Uptake of Radioactive Iodine," Obstetrics \& Gynecology, Vol. 44, 1974, pp. 398-402.

[26] C. Aceves, P. Garcia-Solis, O. Arroyo-Helguera, L. VegaRiveroll, G. Delgado and B. Anguiano, "Antineoplastic Effect of Iodine in Mammary Cancer: Participation of 6Iodolactone (6-IL) and Peroxisome Proliferator-Activated Receptors (PPAR)," Molecular Cancer, Vol. 8, No. 1, 2009, p. 33. doi:10.1186/1476-4598-8-33

[27] F. R. Stoddard II, A. D. Brooks, B. A. Eskin and G. J. Johannes, "Iodine Alters Gene Expression in the MCF7 Breast Cancer Cell Line: Evidence for an Anti-Estrogen Effect of Iodine," International Journal of Medical Sciences, Vol. 5, No. 4, 2008, pp. 189-196. doi:10.7150/ijms.5.189

\section{List of Abbreviations}

ER-Estrogen receptor;

$\mathrm{PR}$ - progesterone receptor;

I- -Iodide;

$\mathrm{I}_{2}$-Iodine;

$\mathrm{T}_{4}$ - thyroxine. 